\title{
Spinning and tumbling of long fibers in isotropic turbulence
}

\author{
Theresa B. Oehmke $\odot,{ }^{1}$ Ankur D. Bordoloi $\odot,{ }^{1}$ Evan Variano, ${ }^{1}$ and Gautier Verhille $\odot^{2, *}$ \\ ${ }^{1}$ Department of Civil and Environmental Engineering, University of Berkeley CA 94720, USA \\ ${ }^{2}$ Aix Marseille Univ, CNRS, Centrale Marseille, IRPHE, F-13013 Marseille, France
}

(Received 16 November 2020; accepted 23 March 2021; published xxxxxxxxxx)

We simultaneously measure both spinning and tumbling components of rotation for long near-neutrally buoyant fibers in homogeneous and isotropic turbulence. The lengths and diameters of the measured fibers extend to several orders of the Kolmogorov length of the surrounding turbulent flow. Our measurements show that the variance of the spinning rate follows a $-4 / 3$ power-law scaling with the fiber diameter $(d)$ and is always larger than the variance of the tumbling rate. This behavior surprisingly resembles that observed previously for sub-Kolmogorov fibers. These observations suggest that long fibers preferentially align with vortex filaments that can be as long as the integral length of turbulence. We compute the Lagrangian timescale and the distribution of both tumbling and spinning that supports this outlook. Our measurements also allow us to quantify the importance of the Coriolis term on the rotational dynamics of fibers in turbulent flows.

DOI: 10.1103/PhysRevFluids.00.004600

\section{INTRODUCTION}

Since 2010, an increasing number of studies have been devoted to the understanding of rotation of anisotropic particles in turbulent flows. The growing interest in this research can be attributed to the numerous applications of such particles found in the environment as well as in industries. The tumbling of elongated fibers is important in paper-making processes. Examples of such applications are also found in polymer processing [1], fiber-reinforced-composite molding [2], turbulent drag reduction strategies [3], etc.

In real applications, most particles are anisotropic ranging from simpler ones, such as rods and discs to much more complex shapes [4]. Considering one of the simplest scenarios of an axisymmetric fiber, the rotation can be decomposed into two motions: the tumbling, which corresponds to the rotation of the axis of symmetry of the particle, and the spinning, which corresponds to the rotation about that axis. The evolution of the variance of the tumbling rate as a function of fiber length $(L)$ has been studied in detail in several experimental and numerical works. These studies show that the variance of the tumbling rate for near-neutrally buoyant fibers scales as $\ell^{-4 / 3}$ when the fiber length is longer than $\sim 10$ Kolmogorov lengths. The typical length scale $\ell$ corresponds to the fiber length $L$ for an aspect ratio $\Lambda=L / d$ larger than $\sim 3[5,6]$. For smaller aspect ratios $\Lambda \in[1 ; 4]$, Bordoloi and Variano [7] proposed that the pertinent length scale is based on the volume of the particle: $\ell \sim\left(d^{2} L\right)^{1 / 3}$. This scaling was shown to be valid for various shapes with similar aspect ratios [8]. When the fiber inertia cannot be neglected, a filtering effect appears to decrease the variance of the tumbling rate $[9,10]$.

Because of the implicit difficulty in resolving both components of rotation, the research heretofore is mainly limited to the tumbling rate. Using refractive-index-matched Particle Image Velocimetry (PIV) and, by analyzing the shape of the ellipse produced by the laser sheet intersecting

\footnotetext{
*gautier.verhille@irphe.univ-mrs.fr
} 
a cylinder, Bordoloi and Variano [7] reported the decomposition of the two components of rotation for cylinders of aspect ratio, $\Lambda=4$. However, since their experiment was limited only to a single aspect ratio, a complete understanding of the mechanism of rotational partitioning is missing.

The problem also bears an important aspect of fluid mechanics that relates the rotational dynamics of anisotropic particles to the velocity gradient tensor in turbulence [4]. Although most studies to date have primarily focused on the dynamics of rigid particles smaller than the Kolmogorov length $(\eta)[4,11-13]$, some have extended this interest to rigid inertial fibers [5-7,9,10] as well as to flexible fibers [14-19]. Previous theoretical and numerical studies on inertialess fibers shorter than the Kolmogorov length $\eta$ have shown that such small particles strongly align with the local vorticity $[11,13]$. As a consequence, small fibers spin more than they tumble $[4,12,20]$. For fibers longer than the Kolmogorov length $\eta$, such preferential sampling of the velocity field has not been investigated in details. Pujara et al. [21] showed numerically that when the fiber length $L$ exits the viscous regime $(L<\eta)$ and enters the inertial regime $(L>\eta)$, the preferential orientation switches from the local vorticity to the most extensional eigenvector of the coarse-grained strain rate tensor. This could suggest that the spinning rate of a long fiber should decrease with length, as the preferential orientation with the vorticity is lost when the fiber length is in the inertial regime. On the contrary, by studying preferential sampling of both flexible and rigid fibers, Picardo et al. [18] showed that long fibers tend to be preferentially trapped within the vortex tubes in turbulence. In that case, the rate of spinning would increase and might exceed that of tumbling.

The goal of this paper is to report direct simultaneous measurements of both spinning and tumbling rates of long inertial fibers in turbulent flows. In the following section, Sec. II, we discuss the experimental apparatus and the postprocessing methods used to compute the two components of rotation. In Sec. III, we present the evolution of the tumbling and the spinning rates of these fibers. We analyze and discuss these results in the context of preferential alignment, fiber inertia, timescale of rotation, and turbulence intermittency in three subsequent subsections. In the final section, Sec. IV, we conclude with a summary of the key findings of this investigation.

\section{EXPERIMENTAL SETUP AND METHODS}

The turbulence is generated by strategically stirring the water filled inside a $60 \mathrm{~cm} \times 60 \mathrm{~cm} \times$ $60 \mathrm{~cm}$ cubic tank. At each corner, an impeller (diameter $=17 \mathrm{~cm}$ ) with eight straight blades of thickness $5 \mathrm{~mm}$ is driven independently using a $1.5 \mathrm{~kW}$ brushless motor. Each impeller is set to rotate at the same frequency but in a chirality opposite to its three nearest neighbors as shown in Fig. 1. The intensity of turbulence inside the tank is set by the impeller frequency between $5-15 \mathrm{~Hz}$. In this configuration, PIV measurements show that the turbulence is homogeneous and isotropic in a cubic subvolume of $\approx 10 \mathrm{~cm} \times 10 \mathrm{~cm} \times 10 \mathrm{~cm}$, centered at the center of the tank. The homogeneity of the turbulence is confirmed by the fact that when dividing the main volume in nine equal subvolumes the statistics of tumbling and spinning are independent of the considered box (up to the statistic convergence of our measurements). The mean flow is also negligible (the kinetic energy of the mean flow is around 100 times smaller than the kinetic energy of the turbulent fluctuations). All the measurements presented in this paper are performed in this region. Each axis of the reference frame points toward a window and the $z$ axis is parallel and opposite to the direction of gravity, cf. Fig. 1.

We use two different fluids (pure water and a mixture of water and Ucon) to vary the kinematic viscosity $v$ and hence the Kolmogorov length $\eta$ and time $\tau$ on wide ranges. We mix Ucon with water at two concentrations (approximately $8 \%$ and $11 \%$ by volume) to increase the liquid viscosity by a factor of 6 or 11 from that of water. At the highest concentration used, the fluid density increases to $\rho_{f}=1.015 \mathrm{~kg} \cdot \mathrm{m}^{-3}$, which is within $2 \%$ of water density. This change of fluid density is relatively small and is assumed negligible in the present paper. For all tested configurations, the viscous boundary layer on the impeller is smaller than the height of the blade. Hence, in this range of viscosity, the forcing is always an inertial forcing and the dissipation rate $\epsilon$ is independent of the kinematic viscosity [22]. The Kolmogorov time and length and the Taylor length in the 
(a)

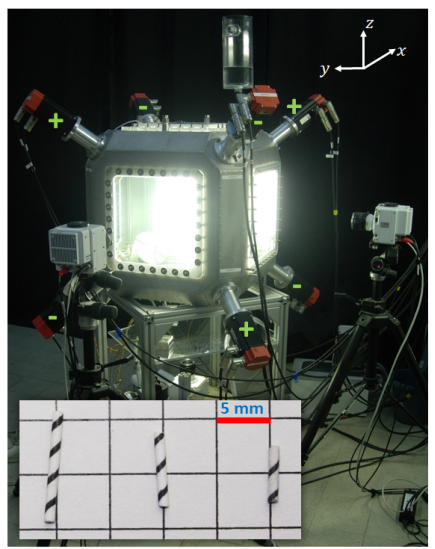

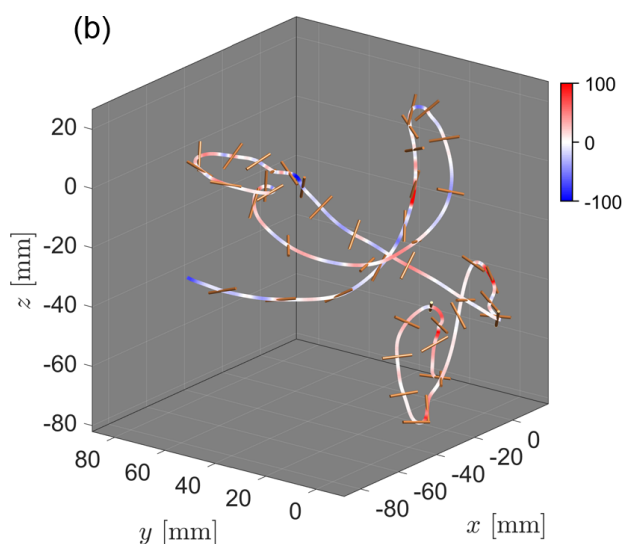

(c)

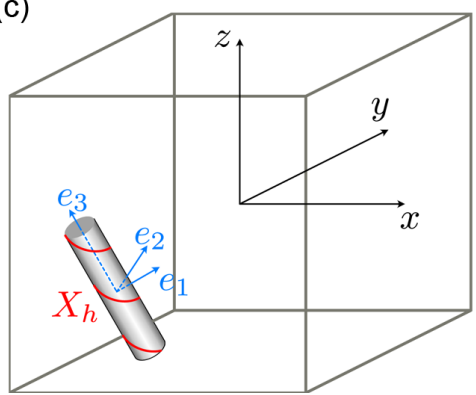

FIG. 1. (a) Photograph of the experimental setup. The cubic tank has a side length of $60 \mathrm{~cm}$. Seven of the eight motors are visible. + or - indicates the direction of the rotation of each impeller. The three cameras are visible along the $x, y$, and $z$ axes. The lightning used here is different from the one used for the experiments for artistic reasons. On the bottom of the image, the three different kinds fibers 10, 7, and $5 \mathrm{~mm}$ from left to right are shown. (b) Example of 3D trajectory of a 10-mm fiber. The color of the trajectory codes the spinning rate of the fiber $\left(\right.$ in s $^{-1}$ ). (c) Sketch representing the different notations used for the 3D reconstruction.

mixture of water and Ucon are determined from the measurement in water and by replacing the kinematic viscosity of water by the one of the mixture. Main statistical quantities of turbulence in the volume of measurement are given in Table I. These values have been validated during our previous studies $[9,23]$ by comparing the evolution of the normalized variance and the correlation time of the tumbling rate, which are in good agreement with other studies.

We use polystyrene fibers with diameter $d=0.93 \mathrm{~mm}$ and density $\rho_{p}=1.04 \mathrm{~kg} . \mathrm{m}^{-3}$, cut to lengths $L=5,7$ or $10 \mathrm{~mm}$. Both the length and the diameter are in the inertial range of turbulence. To measure the spinning, a regular helix is printed on the fiber with a pitch of $2.5 \mathrm{~mm}$ (see Fig. 1). The tumbling Stokes number, quantifying the influence of fiber inertia on the tumbling rate, $\mathrm{St}_{T}=$ $\left(\rho_{p} / \rho_{f}\right)\left(d / \eta_{K}\right)^{4 / 3}(d / L)^{2 / 3}$, defined in Bounoua et al. [9], is always smaller than $2 \times 10^{-2}$, such that

TABLE I. Turbulence properties for the different experiments presented in this paper.

\begin{tabular}{lcccc}
\hline \hline Integral length & Taylor length & Reynolds number & Kolmogorov length & Kolmogorov time \\
$L_{I}$ & $\lambda$ & $R_{\lambda}$ & $\begin{array}{c}\eta_{K} \\
{[\mu \mathrm{m}]}\end{array}$ & $\begin{array}{c}\tau_{K} \\
{[\mathrm{~ms}]}\end{array}$ \\
\hline 7 & {$[\mathrm{~mm}]$} & & $34-434$ & $1.2-17.1$ \\
\hline \hline
\end{tabular}


the inertia of the fiber is negligible at least for tumbling. When the carrying fluid is the mixture of water and Ucon, the fiber was coated with a transparent varnish paint (Luxens) to avoid the dissolution of the ink into the fluid. The layer of the paint was thin enough to neglect the modification of the diameter and of the density. In all cases, fibers are slightly heavier than the carrying fluid. However, the settling velocity $U_{S} \sim\left(\rho_{p}-\rho_{f}\right) d^{2} g / 16 \mu$, where $g$ is the acceleration due to gravity and $\mu$ the dynamic viscosity of the fluid, is at least one order of magnitude smaller than the turbulent fluctuations. Hence, buoyancy effects are negligible here. The volumetric concentration of fibers $\phi$ is small $\left(\phi<10^{-7}\right)$, so interaction among fibers and the modification of turbulence by fibers are negligible.

We image the fibers using three high speed cameras (Phantom VEO 710L) with resolution of 1 MPix triggered simultaneously at a frame rate of 1000-3000 fps. The images are captured through a $50 \mathrm{~mm}$ lens (Zeiss Planar T 1.4/50) mounted on each camera. The fibers are backlit by an LED panel for the camera pointing along the $z$ axis. Two additional LED spot lights of 6600 lumen are used to visualize the pattern printed onto the fiber with the two cameras parallel to the $x$ and $y$ axes.

\section{Postprocessing}

Lagrangian time-series of 3D position and the orientation of each fiber are determined by analyzing the three sets of images. To obtain the 3D reconstruction, we determine the translation vector $(\boldsymbol{T})$ and the rotation matrix $(\boldsymbol{R})$ that transform a virtual fiber initially located at the center of the cube $\left(X_{0}=[0 ; 0 ; 0]\right)$ with its axis of symmetry parallel to the $z$ axis to the location and the orientation of the fiber imaged by each camera. In the fiber frame of reference, the axes of the virtual fiber are denoted as $\left[e_{1}, e_{2}, e_{3}\right]$, such that initially these axes coincide with the laboratory axes (i.e., $e_{1}=e_{x}, e_{2}=e_{y}$ and $e_{3}=e_{z}$ ). We define a set of points along the virtual helix as $X_{h}$.

The cameras are modeled with the classical pinhole model. In this model, a camera is characterized by 11 parameters: the position and the orientation of its frame in the laboratory frame (determined by three angles of rotation), its focal distances, the coordinates of the projection of the pinhole onto the image, and the skew parameter (for details, see, for instance, Refs. [24-26]). These parameters are determined during a calibration process where a sphere is moved to a known set of locations and imaged by the three cameras. The calibration is performed with the fluid inside the cube to take into account the variation of refractive index between the fluid and the air. As the axis of the camera is perpendicular to the viewing window, the distortions of the ray light due to the refraction at the fluid/plexiglass/air interfaces can be neglected [27]. We also ensure that the optical distorsions of the lenses are negligible.

Using homogeneous coordinates, as is classically done in computer vision, the coordinates of a set of points after a rotation by a matrix $\boldsymbol{R}$ and a translation by a vector $T$ is given by

$$
Q_{f} \propto\left(\begin{array}{cccc} 
& \boldsymbol{R} & & T \\
& & & \\
0 & 0 & 0 & 1
\end{array}\right) Q_{0},
$$

where $Q_{0}=\left(x_{0}, y_{0}, z_{0}, 1\right)^{\dagger}$ and $Q_{f}=\left(x_{f}, y_{f}, z_{f}, 1\right)^{\dagger}$ are the homogeneous coordinates of a set of points corresponding to the Cartesian coordinates $\left(x_{i}, y_{i}, z_{i}\right)$ before $(i=0)$ and after $(i=f)$ the rotation/translation [24-26]. We reconstruct each fiber by determining a translation vector $T$ and a rotation matrix $\boldsymbol{R}$ as described below.

The rotation matrix can be decomposed into two terms: $\boldsymbol{R}=\boldsymbol{R}_{T} \boldsymbol{R}_{S}$, where $\boldsymbol{R}_{S}$ is the rotation matrix for spinning (that is, rotation parallel to the $z$ axis), and $\boldsymbol{R}_{T}$ is the rotation matrix for tumbling. Each matrix is determined independently in two steps. First, we characterize the fiber based on a position vector $T_{0}$ and an orientation matrix $R_{T, 0}$ determined from a Shape from Silouhette algorithm, also known as the convex hull volume method [28]. In this method, a fiber is reconstructed as a set of voxels. $T_{0}$ is determined from the center of mass of the group of voxels, and $R_{T, 0}$ is the rotation matrix which rotates $e_{z}$ into the vector $n$ connecting the extremities of the 

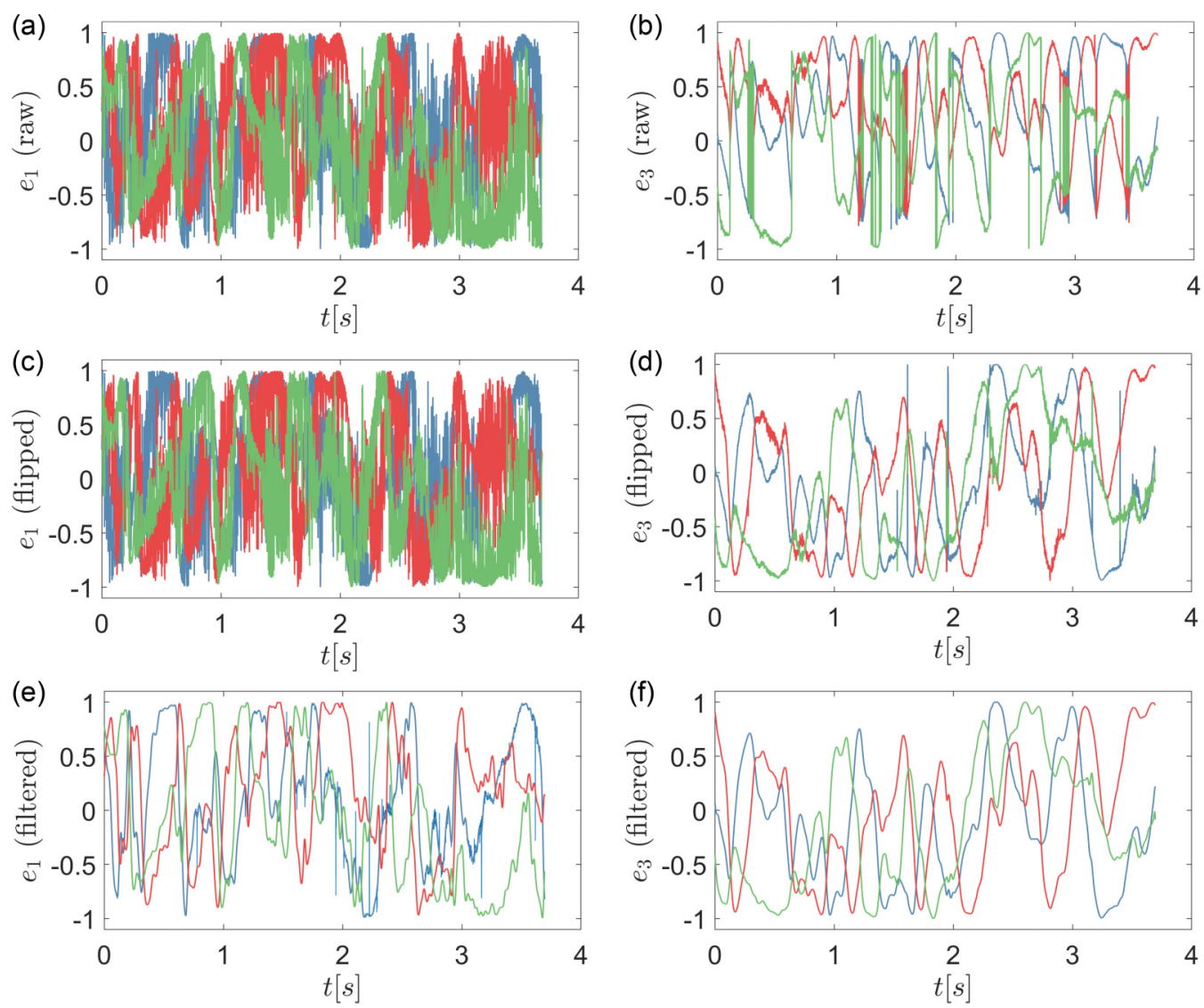

FIG. 2. Time evolution of the three components ( $x$ blue, $y$ red, $z$ green) of $e_{1}$ (left) and $e_{3}$ (right) for the raw trajectory (top), after the flipping step (middle), and the final smoothing process (bottom).

group of voxels. In the second step, the position and the orientation of each fiber is optimized through an optimization process similar to Bounoua et al. [9]. The cost function to be minimized during the optimization process is the distance between the projection of the reconstructed fiber and the location of the real fiber detected on each image.

The rotation matrix of spinning $\boldsymbol{R}_{S}$ is determined similarly by minimizing the cost of projection of the virtual helix $\left(X_{h}\right)$ onto the two images from the cameras parallel to the $x$ and $y$ axes. To perform this optimization, only the points of the helix visible to each camera should be considered. These points can be selected knowing the parameters of the camera and the position and the orientation of the fiber. At the end of the optimization process, we use the Rodrigues' rotation formula, which allows conversion of the rotation matrix $\boldsymbol{R}$ around an axis $k_{r}$ by an angle $\theta$, into a Rodrigues's vector $x_{r}=\theta k_{r}$, and vice versa. We store the translation vector $T$ and the Rodrigues's rotation vector $x_{r}$ for further analysis.

Once all images are postprocessed, we extract the trajectories of individual fibers using the method of the nearest-neighbor described in Ouellette et al. [29]. As the concentration of fiber is very low $\left(<1 \times 10^{-7}\right)$ and the camera acquisition rate is high enough, only the criterion of fiber-fiber distance is used. If several fibers along the trajectory satisfy this criterion, the fiber with the orientation closest to that of the previous time stamp is selected as a candidate for the trajectory. Figures 2(a), 2(b) show the time evolution of the three components of $e_{1}$ and $e_{3}$ vectors in the laboratory frame, respectively, for a sample raw trajectory. 
In Fig. 2(b), the peaks on the trajectory of $e_{3}$ are due to the ambiguity in the direction (positive versus negative) of the axis of symmetry $\left(e_{3}\right)$ between two successive time stamps. We overcome this ambiguity via a consistency check, such that the direction of $e_{3}$ of the fiber is flipped if the dot product between $e_{3}(t)$ and $e_{3}(t-\mathrm{d} t)$ is negative (see Figs. 2(c), 2(d)).

As the thickness of the helix is of the order of one or two pixels, the amplitude of the noise is higher on $e_{1}(t)$ and $e_{2}(t)$ than on $e_{3}(t)$, as seen in the middle panel of Fig. 2. One approach would be to filter $e_{i}(t)$ for $i=1,2$, and then to compute the statistics on the filtered data $e_{i}^{f}(t)$. This approach, however, does not guarantee that the three vectors $e_{1}, e_{2}$, and $e_{3}$ form an orthogonal basis. To overcome this difficulty and increase the precision of the measurement, the dynamics of $e_{1}(t)$ are filtered by applying a Gaussian filter whose standard deviation is less than $2 \tau_{K}$. The amplitude of noise being very small for $e_{3}(t)$, at each time step we determine an optimal Rodrigues vector that minimizes the distance between $e_{1}(t)$ and $e_{1}^{f}(t)$ with a constraint that $e_{3}(t)$ remains unchanged. Finally, the temporal derivative of the different vectors is computed by fitting locally the trajectory with spline function of order 3 (Figs. 2(e) and 2(f)). The number of points used to compute the spline corresponds to the standard deviation of the Gaussian kernel used to filter the data $e_{1}(t)$. We have checked that the different results presented here do not depend on this value [30].

To obtain convergence in the statistics, only trajectories longer than 100 frames (between 6 and 30 Kolmogorov time, depending on the rotation frequency and the fluid viscosity) are used. The longest trajectory is of the order of several seconds for each case, representing several integral times.

The tumbling is determined from the variation of the orientation vector $e_{3}$ using a central difference scheme $\left(\dot{e}_{3}\right)$ for each fiber. In the laboratory reference frame, the tumbling vector $\Omega_{T}$ can be computed by solving

$$
\dot{e}_{3}=\Omega_{T} \times e_{3} \quad \text { and } \quad \Omega_{T} \cdot e_{3}=0 .
$$

Contrary to the tumbling rate, the spinning rate cannot be determined directly from the temporal evolution of $e_{1}$ or $e_{2}$, as they depend on both tumbling and spinning. Therefore, the determination of the spinning rate requires the removal of the contribution from tumbling. The rotation matrix $\boldsymbol{R}_{T}(t)$ related to tumbling is given by the evolution of $e_{3}(t)$ as

$$
e_{3}(t)=\boldsymbol{R}_{T}(t) e_{3}(0),
$$

enforcing that the rotation axis associated to this matrix is perpendicular to both $e_{z}$ and $e_{3}$. Knowing this matrix $\boldsymbol{R}_{T}$, one can define the spinning rotation matrix $\boldsymbol{R}_{S}=\boldsymbol{R}_{T}^{-1} \boldsymbol{R}_{\text {opt }}$. The spinning rate $\left(\Omega_{S}\right)$ is then determined from the spinning vector $e_{S}(t)=\boldsymbol{R}_{S}(t) e_{1}(0)$ in the fiber frame, using

$$
\dot{e}_{S}=\Omega_{S} \times e_{S} .
$$

Another possibility is to compute directly the total rotation vector $\Omega$ from the temporal evolution of the fiber frame: $\dot{e}_{i}=\Omega \times e_{i}$ for $i=1,2,3$. The spinning vector corresponds then the third component of $\Omega$ and the tumbling to the two first components. We checked that the presented results are comparable with both methods. However, the amplitude of the noise was smaller with the first method.

\section{RESULTS AND DISCUSSION}

\section{A. Variances of tumbling and spinning rates}

Figure 3 presents the normalized variance of the tumbling rate $\left(\left\langle\Omega_{T} \Omega_{T}\right\rangle \tau_{K}^{2}\right)$ with respect to the normalized fiber length $(L / \eta)$. These results are compared with those from Parsa and Voth [5] who studied similar cases. Results from both sets of experiments overlap and show an evolution of the variance of the tumbling rate, as $\left\langle\Omega_{T} \Omega_{T}\right\rangle \sim(L / \eta)^{-4 / 3} \tau_{K}^{-2}$, in agreement with the slender body prediction. In this model, fiber inertia is neglected so the global torque $\Gamma$ applied on the fiber is equal to 0 . Considering only the viscous torque [9], the total torque applied on the fiber can be 


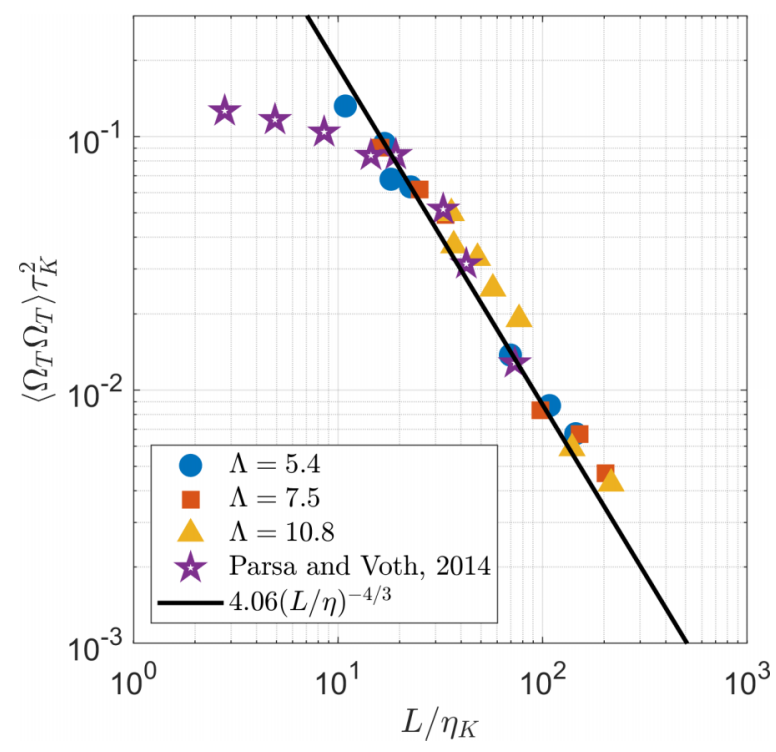

FIG. 3. Dimensionless variance of tumbling rate of inertial fibers against dimensionless fiber length. The prefactor in the $-4 / 3$ power-law is obtained from a least-squares fit of the experimental data.

modeled by

$$
\Gamma=\int_{-L / 2}^{L / 2} \mu u_{g} \times s d s=0,
$$

where $\mu$ is the dynamical fluid viscosity, $u_{g}=u_{f}-v$ is the slipping velocity, with $u_{f}$ and $v$ denoting the fluid and the fiber velocities, respectively. The notation $s$ represents the curvilinear coordinate along the fiber whose origin is at the center of mass. As the fiber is rigid, the velocity of the fiber in the frame attached to the fiber can be written $v=s \Omega_{T}$. Hence, the average tumbling rate from Eq. (5) scales as

$$
\Omega_{T} \sim \frac{1}{L^{3}} \int_{-L / 2}^{L / 2} u_{f} \times s d s
$$

In the framework of Kolomogorov 1941 (K41) theory, only the structure whose length is comparable to the fiber length contributes to the torque. The fluid velocities $\left(u_{L}\right)$ at this scale are constant over the fiber length so the integral in Eq. (5) vanishes. This integral also vanishes for velocities at a scale much smaller than the fiber length as they are not correlated along the fiber. Hence, the integral reduces to the slender body scaling, $\left\langle\Omega_{T} \Omega_{T}\right\rangle \sim\left(u_{L} / L\right)^{2} \sim \epsilon^{2 / 3} L^{-4 / 3}$.

Following this framework, spinning is forced by the local vorticity at the scale of the diameter: $u_{d}(s) \sim d \nabla_{d} u_{f}(s)$, where $\nabla_{d}$ is a coarse grained gradient at scale $d$. The spinning rate vector should therefore scale as

$$
\Omega_{S} \sim \frac{1}{L d} \int_{-L / 2}^{L / 2} u_{d}(s) d s .
$$

As the increment of velocity $u_{d}$ is assumed to be correlated on scale $d \ll L$, the integral should vanish for long aspect ratios. Hence, the spinning rate is expected to be null or at least much smaller than the tumbling rate $\left(\Omega_{T}\right)$ for long fibers. Below, we report our experimental measurements of the spinning rate and test this assumption.

Figure 4(a) shows the normalized variance of the spinning rate $\left(\left\langle\Omega_{S} \Omega_{S}\right\rangle \tau_{K}^{2}\right)$ as a function of the normalized fiber length $\left(L / \eta_{K}\right)$. The global trend for the three aspect ratios $(\Lambda)$ is a decrease of 

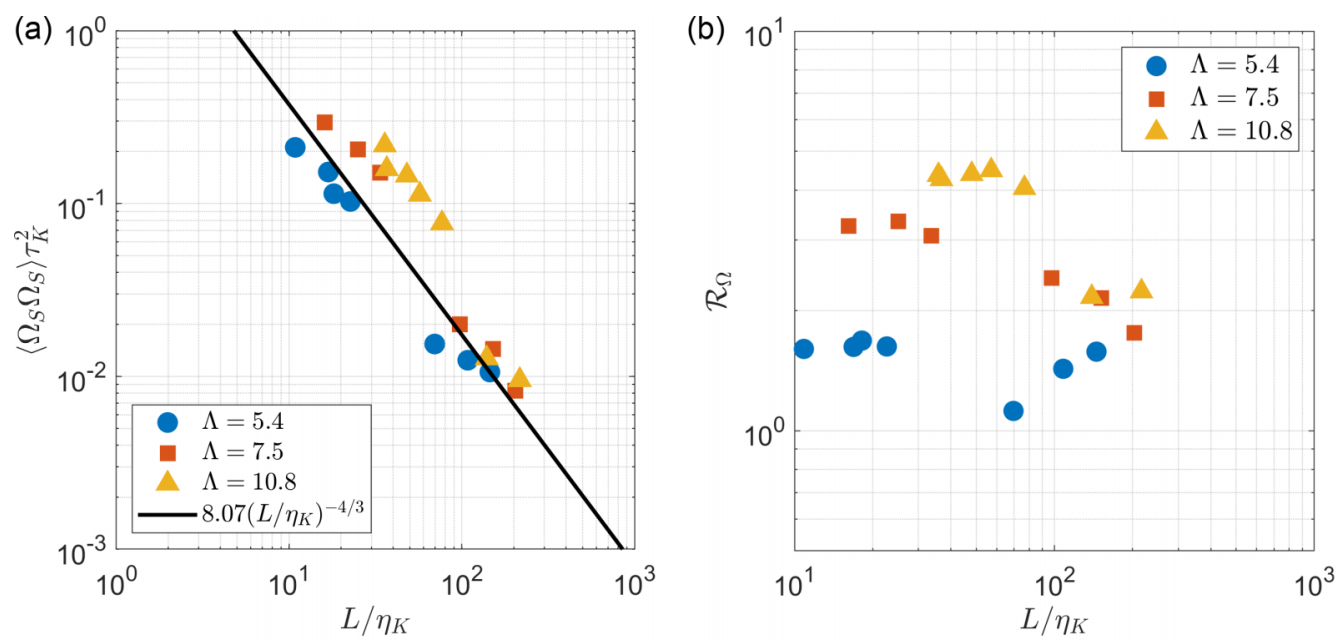

FIG. 4. (a) Dimensionless variance of spinning rate and (b) the ratio between the variances of spinning and tumbling rates with respect to dimensionless fiber length.

the spinning rate with increasing the normalized fiber length. However, compared to $\left\langle\Omega_{T} \Omega_{T}\right\rangle \tau_{K}^{2}$ (see Fig. 3), the data points for $\left\langle\Omega_{S} \Omega_{S}\right\rangle \tau_{K}^{2}$ are largely scattered about the $-4 / 3$ power-law fit. This suggests that the fiber length is not or not the only controlling parameter of the spinning rate. This is even more evident in the ratio $\mathcal{R}_{\Omega}$ between the variances of spinning and tumbling $\mathcal{R}_{\Omega}=\left\langle\Omega_{S} \Omega_{S}\right\rangle /\left\langle\Omega_{T} \Omega_{T}\right\rangle$ of rotation plotted against the normalized fiber length [see Fig. 4(b)]. $\mathcal{R}_{\Omega}$ increases with $\Lambda$, showing the importance of the fiber diameter $(d)$. Figure 4(b) also shows that the variance of spinning rate is always larger than that of the tumbling rate. This contradicts the expectation from K41 theory discussed in Sec. III A.

These observations raise two important questions. First, what is the mechanism of forcing behind the spinning of long fibers in turbulence? Second, what is the consequence of the amplitude of the spinning rate on the global rotation dynamics of fibers in turbulence? In earlier studies [4,9], the Coriolis term $\Omega \times I \Omega$ was always neglected when modeling the tumbling rate of long fibers. Our current observation urges an investigation related to the validity of this assumption. We address these two questions in the following two sections.

\section{B. Spinning rate and preferential alignment}

We examine here two mechanisms that could possibly induce spinning in a long fiber. In the first, the spinning is forced by the coarse-grained vorticity at the scale of the fiber length $L$. This scenario is incompatible with the results of Pujara et al. [21], where they showed that the alignment of fibers with vorticity decreases when fiber length is increased. Moreover, this scenario requires the spinning rate to scale with the fiber length $L$, which is not compatible with the scattering observed in Fig. 4(a). The second scenario postulates a preferential alignment with structures that imposes a velocity difference at the scale of the fiber diameter coherent along the fiber length as proposed in Picardo et al. [18]. To test this scenario, we show the evolution of the normalized variance of the spinning rate as a function of the normalized fiber diameter in Fig. 5(a). Results indicates that the scattering of the data points is reduced compared to that in Fig. 4(a). This suggests that the spinning is indeed due to the shear at the scale of the fiber diameter:

$$
\left\langle\Omega_{S} \Omega_{S}\right\rangle \sim\left(u_{d} / d\right)^{2} \sim(d / \eta)^{-4 / 3} \tau_{K}^{-2} .
$$



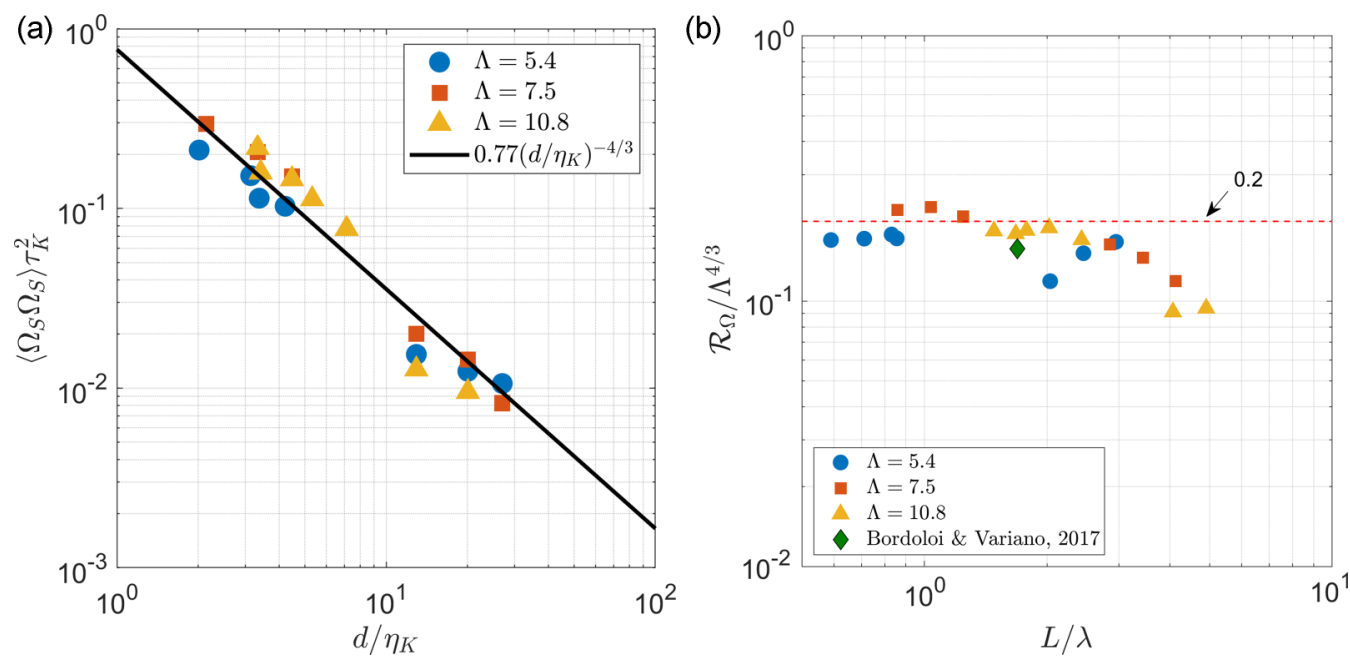

FIG. 5. (a) Dimensionless variance of spinning rate as a function of the dimensionless fiber diameter and (b) a compensated ratio between the variances of the spinning and the tumbling rate $\left(\mathcal{R}_{\Omega} / \Lambda^{4 / 3}\right)$ against fiber length $L$ normalized by Taylor length $\lambda$.

The evolution of the spinning rate with the fiber diameter discussed above cannot be explained only on the basis of the classical K41 approach. Indeed, within this framework, a structure of size $\ell$ is correlated over the size of order $\ell$. Therefore, the integral over a length greater than $\ell$ vanishes, as $\left\langle u_{\ell}\right\rangle=0$. This is obviously not the case here. The observed scaling of the spinning rate implies that fibers might be preferentially aligned with elongated structures where transverse increments of velocity are correlated over a longer length scale. In turbulent flows, such structures exist typically as the filaments of coherent vorticity as first evidenced by Douady et al. [31]. These coherent structures can be very long, up to the integral length of the flow but are generally twisted and randomly oriented. The forcing of the spinning is then only possible as long as the fiber length is smaller or of the order of the correlation length of the axial vorticity of these filaments. Jiménez and Wray [32] showed numerically that this correlation length is given by the Taylor length scale $\lambda$. Such preferential alignment of elongated particles with coherent vortices has also been recently reported by Picardo et al. [18] for flexible fibers. To relate our work to that of Pujara et al. [21], our results suggest that the rotational dynamic of fibers is not only governed by a coarse grained velocity field at the scale of the fiber length $L$ but by a bandpass filtered velocity field bounded by the fiber length $L$ and diameter $d$ [33]. At a first-order approximation, the normalized fiber length $\left(L / \eta_{K}\right)$ governs the tumbling and the normalized diameter $\left(d / \eta_{K}\right)$ governs the spinning.

We examine this hypothesis in Fig. 5(b), which shows the evolution of the compensated ratio $\mathcal{R}_{\Omega} / \Lambda^{4 / 3}$ with respect to fiber length $(L)$ normalized by the Taylor length scale $(\lambda)$. Results show that $\mathcal{R}_{\Omega} / \Lambda^{4 / 3}$ is nearly constant and equal to 0.2 up to $L \sim 2 \lambda$, after which it continues to decrease. This supports the argument that the forcing of the spinning is due to coherent structures whose correlation length scales with the Taylor scale.

Figure 6(a) shows the normalized variance of total rotation rate, $\langle\Omega \Omega\rangle \tau_{K}^{2}=$ $\left(\left\langle\Omega_{S} \Omega_{S}\right\rangle+\left\langle\Omega_{T} \Omega_{T}\right\rangle\right) \tau_{K}^{2}$ with respect to the normalized fiber diameter $\left(d / \eta_{K}\right)$. Combining the scalings of the variances of spinning and tumbling rates with respect to fiber diameter and length, the variance of total rotation rate can be expressed as

$$
\begin{aligned}
\langle\Omega \Omega\rangle_{1} \tau_{K}^{2} & =\left\langle\Omega_{S} \Omega_{S}\right\rangle \tau_{K}^{2}+\left\langle\Omega_{T} \Omega_{T}\right\rangle \tau_{K}^{2} \\
& =C_{S}\left(d / \eta_{K}\right)^{-4 / 3}\left(1+C_{T} / C_{S} \Lambda^{-4 / 3}\right) .
\end{aligned}
$$



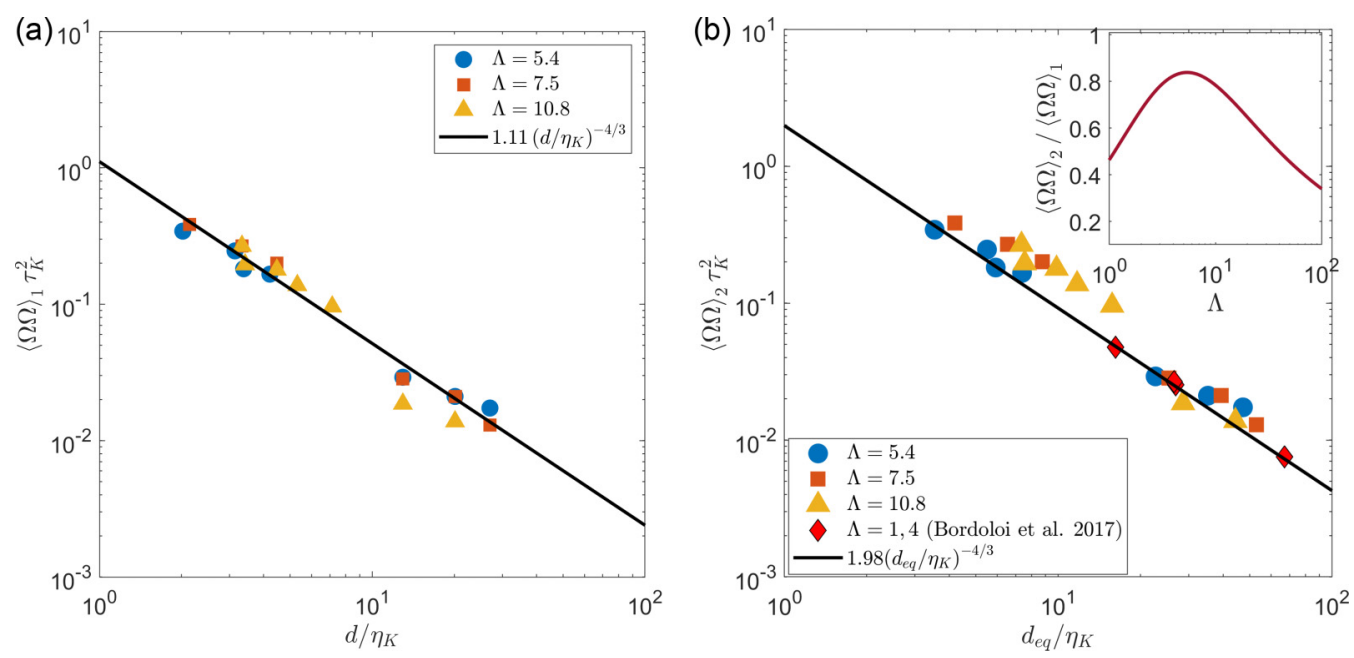

FIG. 6. Dimensionless variance of total rotation rate against dimensionless (a) fiber diameter based on Eq. (9), (b) spherical volume equivalent diameter based on Eq. (10) and the evolution of the ratio between the two variances as a function of aspect ratio in the inset.

Here, $C_{T}=4.06$ and $C_{S}=0.77$ are the two constants of proportionality corresponding to the best fit shown on Figs. 3 and 5(a). Equation (9) suggests that $\langle\Omega \Omega\rangle$ follows a $-4 / 3$ power-law with respect to $d / \eta_{K}$, and the prefactor depends on the aspect ratio $\Lambda$. For the three aspect ratios considered in this paper, the prefactor varies between 1.1 and 1.63.

Bordoloi and Variano [7] found empirically that the evolution of the variance of the total rotation is well described by a power law $\langle\Omega \Omega\rangle \sim \tau_{K}^{-2}\left(d_{e} q / \eta_{K}\right)^{-4 / 3}$, where $d_{\mathrm{eq}} \sim d \Lambda^{1 / 3}$ is the volume equivalent spherical diameter. This relation can be rewritten as

$$
\langle\Omega \Omega\rangle_{2} \tau_{K}^{2}=C\left(d / \eta_{K}\right)^{-4 / 3} \Lambda^{-4 / 9} .
$$

Figure 6(b) shows also a relatively good agreement to the $d_{\mathrm{eq}} / \eta_{K}$ scaling for the aspect ratios $(\Lambda=$ $5.4,7.5,10.8)$ considered in these studies and the aspect ratio presented in Bordoloi and Variano [7] $(\Lambda=1,4)$. The solid line in this plot shows the power law fit $1.98\left(d_{\mathrm{eq}} / \eta_{K}\right)^{-4 / 3}$ proposed in Bordoloi and Variano [7]. It is in better agreement with low aspect ratio $(\Lambda=5.4)$ data sets of our experiment. The equivalence of these two scaling laws is captured by the ratio $\langle\Omega \Omega\rangle_{2} /\langle\Omega \Omega\rangle_{1}$ shown in the inset of Fig. 6(b). For the $\Lambda$ values considered in these studies, the ratio between the two variances is close to 1 , such that $\langle\Omega \Omega\rangle_{2} /\langle\Omega \Omega\rangle_{1}$ ranges between $0.5-0.84$ for $\Lambda=1-10$. For larger aspect ratios, we expect that the scaling previously proposed by Bordoloi and Variano [7] underestimates the total rotation $\langle\Omega \Omega\rangle$.

\section{Lagrangian timescales and intermittency}

Given that the variance of the spinning rate of a fiber scales with the fiber diameter $\left(d / \eta_{K}\right)$ and not the fiber length $\left(L / \eta_{K}\right)$, the goal here is to probe if such scaling also exists for the correlation time of the spinning rate. Following the method described in Bordoloi et al. [23], we compute two timescales, namely, the zero-crossing time $\left(\tau_{0}\right)$ and the integral time $\left(\tau_{i}\right)$, based on the mean autocorrelation of the spinning and the tumbling rates from approximately 1500 trajectories. The details of this computation can be found in Bordoloi et al. [23].

Figure 7(a) recovers the trend observed in Bordoloi et al. [23] and shows that the evolution of the normalized correlation times $\left(\tau_{0, t} / \tau_{K}\right.$ and $\left.\tau_{i, t} / \tau_{K}\right)$ of the tumbling rate with the normalized fiber length $\left(L / \eta_{K}\right)$ collapses on a power-law, $\tau_{i} / \tau_{K} \sim \tau_{0} / \tau_{K} \sim\left(L / \eta_{K}\right)^{2 / 3}$. This result reemphasizes that the fiber length $\left(L / \eta_{K}\right)$ characterizes not only the variance but also the Lagrangian timescale of the 

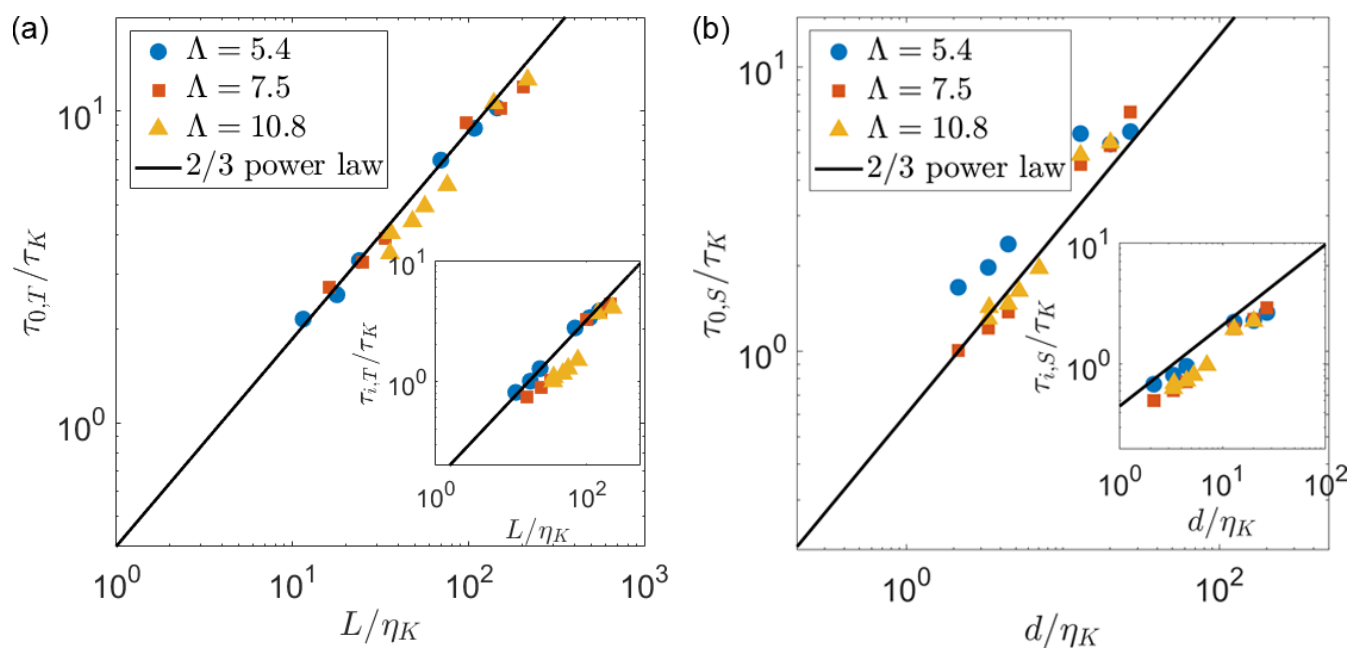

FIG. 7. Evolution of the zero-crossing time for (a) tumbling rate as a function of the normalized fiber length $\left(L / \eta_{K}\right)$ and (b) spinning rate as a function of the normalized fiber diameter $\left(d / \eta_{K}\right)$. Each inset shows the evolution of the integral correlation timescale for the respective component of rotation.

tumbling rate, and that the fiber diameter $\left(d / \eta_{K}\right)$ has no significant role when $S t_{T}<1$. A similar $2 / 3$ power-law scaling is recovered for the normalized correlation times $\left(\tau_{0, s} / \tau_{K}, \tau_{i, s} / \tau_{K}\right)$ of the spinning rate when plotted with respect to the normalized fiber diameter $\left(d / \eta_{K}\right)$ [see Fig. 7(b)]. We do not observe any systematic deviation from the power-law scaling in the correlation timescales of the spinning rate for all tested diameters, unlike the correlation timescales of tumbling rates in Bordoloi et al. [23], which depend on a tumbling Stokes number. Nonetheless, this result confirms that the normalized fiber diameter is an important length scale of the coherent structures that force spinning.

Figures 8(a) and 8(b) present the probability density function (PDF) of the tumbling and the spinning rates, respectively. Each distribution is mean centered and normalized by the rms of the respective component. The mean spinning and tumbling rates are close to zero and much smaller than the respective variance. The distributions are independent of their Cartesian components and, hence, only their vertical component is shown for tumbling.

In the range of fiber sizes tested here, we do not observe a significant effect of the fiber size on the shape of the PDF for both tumbling and spinning within the error bar of our measurements. For the tumbling rate, the PDFs are symmetric and they show exponential decay. This shape is compatible with the one observed by Parsa and Voth [5], who presented the PDF of the norm of tumbling rate. Its kurtosis, $\mathcal{F}_{x}=\left\langle(x-\langle x\rangle)^{4}\right\rangle /\left(\langle x-\langle x\rangle)^{2}\right\rangle^{2}$, is nearly constant within the error bar of our measurements with a mean value $\left\langle\mathcal{F}_{T}\right\rangle=7.7 \pm 0.2$, cf. Fig. 8(c). By contrast, the PDFs of spinning rate are relatively wider for small rotation rates, with a sharp decay appearing at spinning rates larger than $\sim 3 \Omega_{S}^{\text {rms }}$. This is accompanied by the corresponding values of kurtosis smaller than those for tumbling rates as can be seen in Fig. 8(c). This might seem contradictory with our previous interpretation that spinning is forced by smaller scales than tumbling. Indeed, turbulence intermittency is responsible for the broadening of the PDF of the velocity increments $\delta_{l} u$ with decreasing length scale $\ell$ [34]. We interpret the small value of the kurtosis of spinning rate based on the correlation of the forcing along the fiber length. The structures responsible for the tails of the PDF of velocity increment correspond to high intensity structures. The size of coherent structures is known to decrease when their intensity increases [35,36]. At some point, their size becomes smaller than the fiber length and they can no longer contribute statistically to spinning. This is supported by Fig. 8(c), where the flatness of the spinning is constant for long fibers $L \gtrsim 2 \lambda$ but increases when 

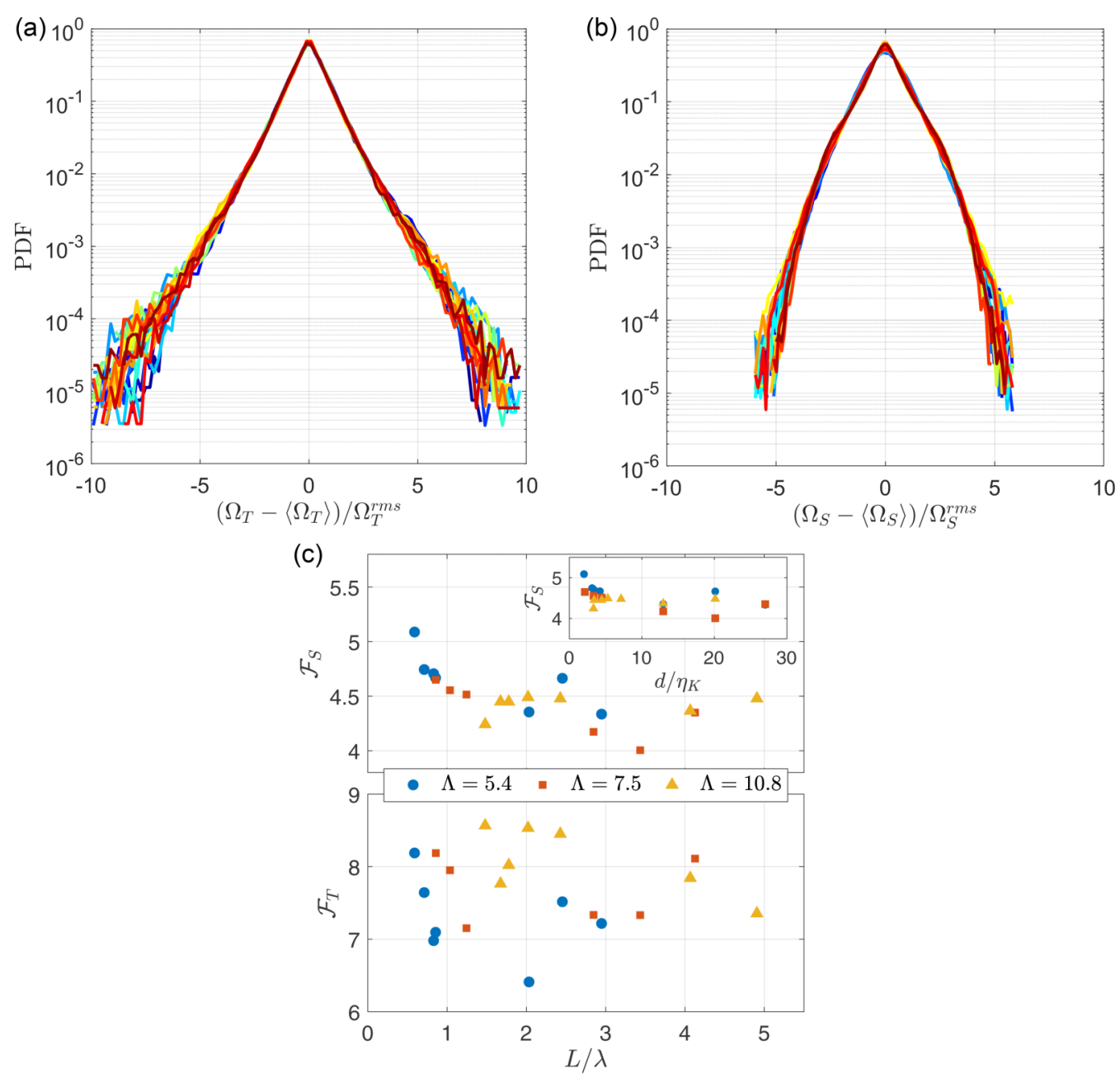

FIG. 8. PDF of mean-subtracted (a) tumbling and (b) spinning rates normalized by their respective rms for fibers of various lengths and diameters. The color-scheme varies from blue to red with increasing $L / \eta_{K}$ and $d / \eta_{K}$ for tumbling and spinning, respectively. (c) Evolution of the kurtosis of spinning (top) and tumbling (bottom) rates as a function of the fiber length normalized by the Taylor length $\lambda$. The inset shows the evolution of the kurtosis of spinning rate as a function of the normalized diameter $d / \lambda$.

the fiber length is decreased for $L \lesssim 2 \lambda$. Therefore, we expect that the shape of the PDF of spinning rate will be closer to the shape of the distribution of the velocity increments at small scales when the fiber length is decreased.

\section{Rotation rate and fiber inertia}

Finally, we examine the validity of the classical assumption of neglecting fiber inertia in estimating the rotational dynamics of fiber. The general equation of conservation of the angular momentum is

$$
\frac{\partial I \Omega}{\partial t}+\Omega \times I \Omega=\Gamma
$$


Ideally, it is necessary to compare each term of the left-hand side of the equation to the global torque on the fiber. This requires measuring the flow field around the fiber, which is beyond the scope of this paper. Therefore, we will use the previous scaling laws to estimate each term.

For the temporal term $\partial_{t} I \Omega$, we define a spinning Stokes number comparing the relaxation time of spinning $\tau_{S} \sim I_{S} / \mu L d^{2}$, where $I_{S}$ is the moment of inertia for spinning, to the forcing timescale $\tau_{d} \sim d / u_{d}$ (see Bounoua et al. [9] for an equivalent definition of the tumbling Stokes number). As $I_{S}=\pi \rho_{p} d^{4} L / 32$ for a homogeneous fiber, the spinning Stokes number scales as

$$
\mathrm{St}_{S}=\frac{\tau_{S}}{\tau_{d}} \sim \frac{\pi}{32} \frac{\rho_{p}}{\rho_{f}}\left(\frac{d}{\eta_{K}}\right)^{4 / 3} .
$$

The spinning component of the second inertial term $\Omega \times I \Omega$ is null for a homogeneous cylindrical fiber. This is not the case for tumbling and this term scales as $\left(I_{S}-I_{T}\right) \Omega_{S} \Omega_{T}$, where $I_{T}$ is the moment of inertia for tumbling. For fibers with $I_{S} / I_{T} \sim(d / L)^{2} \ll 1$, the leading order term therefore scales as $I_{T} \Omega_{S} \Omega_{T}$. This term, compared to the viscous torque for tumbling defined Eq. (6), is negligible if

$$
\Omega_{S} \tau_{T} \ll 1
$$

Here $\tau_{T}$ is the relaxation time of tumbling, defined by the balance of the viscous relaxation term $\int \mu L \Omega_{T} \times s d s$ and the temporal term, and scales as $\tau_{T} \sim I_{T} / \mu L^{3}$ [9]. Invoking the scaling for the spinning rate as $\Omega_{S} \sim u_{d} / d \sim\left(d / \eta_{K}\right)^{-2 / 3} \tau_{K}^{-1}$ into Eq. (13), we can show that this term is negligible as long as $\mathrm{St}_{S}$ is small. In this study, for $\mathrm{St}_{S}$ between 0.25 and 8.2, we do not observe any influence of the fiber inertia on the evolution of the variance of spinning rate, the corresponding correlation time, and the PDF. This suggests that the threshold in $\mathrm{St}_{S}$ is at least one order of magnitude higher than unity, which can be compensated by introducing a prefactor to Eq. (12). Measuring this prefactor deserves further investigation, which has to be done in a setup where the separation of scales between the fiber length scales and the flow length scales satisfy $\eta_{K} \lesssim d<L \ll \lambda$ to remove the influence of the correlation of the forcing along the fiber length. This separation of scales is beyond our current setup with the fibers used in this study.

Nonetheless, an important message that falls out of the above analysis is that, contrary to the tumbling Stokes number, the spinning Stokes number does not depend on the aspect ratio of the fiber and can be significantly large even for a slender body if $d \gg \eta$ [see Eq. (12)]. Therefore, for fibers with $d>\eta_{K}$ and $\Lambda \gg 1$, although the temporal inertial term $\partial_{T} I_{T} \Omega_{T}$ can be ignored in Eq. (13), the second inertial term that couples the tumbling and the spinning motions cannot always be neglected.

\section{CONCLUSION}

We experimentally resolve both components of rotation (spinning and tumbling) of inertial fibers $\left(L \gg \eta_{K} ; d>\eta_{K}\right.$ ) in homogeneous isotropic turbulence. Our measurements show that fibers tend to spin more than to tumble. We show that the variance of the spinning rate follows a power law scaling with respect to the fiber diameter, such that $\left\langle\Omega_{S} \Omega_{S}\right\rangle \tau_{K}^{2} \sim\left(d / \eta_{K}\right)^{-4 / 3}$. This contradicts the classical view based on K41 theory, where the spinning rate of an inertial fiber is considered negligible compared to the tumbling rate. This scaling implies that fibers are preferentially trapped within elongated coherent structures where the transverse increments of velocity are correlated over lengths of the order of Taylor scale of turbulence. We show the importance of the fiber aspect ratio $(\Lambda=$ $L / d)$ via a rescaled ratio $\left(\left\langle\Omega_{S} \Omega_{S}\right\rangle / \Lambda^{4 / 3}\left\langle\Omega_{T} \Omega_{T}\right\rangle\right)$ between the variances of the spinning and the tumbling rates. For a fiber shorter than a few Taylor scales $(L \lesssim 2 \lambda)$, this ratio is nearly constant but decreases rapidly with increasing fiber length. In the future, it would be useful to extend this study to oblate anisotropic particles, such as discs, to examine if the major axes of all anisotropic inertial particles tend to align with the vorticity, similar to sub-Kolmogorov scale particles $[4,13]$. 
Besides, it would also be interesting to study such phenomena for flexible fibers that can conform to the topology of a vortex tube, in the vein of Picardo et al. [18].

In addition, we compute the Lagrangian timescales of spinning and tumbling by analyzing the autocorrelation of the respective components. Both timescales follow the scaling $\tau_{S / T} \sim\left(l / \eta_{K}\right)^{-2 / 3}$, where $l=L$ and $d$ for tumbling and spinning, respectively. We do not observe any obvious deviation from this scaling for spinning even for high Stokes number $\mathrm{St}_{S} \sim 8$. Further, the PDF of spinning rate shows suppression of extreme events beyond $3 \Omega_{S}^{\text {rms }}$, leading to a smaller flatness factor $\left(\mathcal{F}_{S}<\mathcal{F}_{T}\right)$. We hypothesize that this result is due to the lengthwise decorrelation of local forcing responsible of the stronger spinning events. From a modeling point of view, our results show that an inertial fiber in turbulence experiences a velocity field smoothed by a bandpass filter whose length scales are given by its length (related to tumbling motion) and its diameter (related to the spinning motion).

The measurement of the spinning rate also allows us to estimate the importance of the coupled inertial term in the rotation equation which was generally assumed negligible in earlier studies of fiber rotation in turbulence. We show that this assumption should hold only when the spinning Stokes number $\mathrm{St}_{S}$ is small enough.

\section{ACKNOWLEDGMENTS}

This work was carried out in the framework of FlexFiT Project No. ANR-17-CE30-0005-01 funded by the French National Research Agency (ANR).

[1] L. Jarecki, S. Blonski, A. Blim, and A. Zachara, Modeling of pneumatic melt spinning processes, J. Appl. Polym. Sci. 125, 4402 (2012).

[2] S. Yashiro, H. Sasaki, and Y. Sakaida, Particle simulation for predicting fiber motion in injection molding of short-fiber-reinforced composites, Composites Part A: Appl. Sci. Manufacturing 43, 1754 (2012).

[3] W. D. MCCOMB and K. T. J. CHAN, Drag reduction in fibre suspensions: Transitional behavior due to fibre degradation, Nature 280, 45 (1979).

[4] G. A. Voth and A. Soldati, Anisotropic particles in turbulence, Annu. Rev. Fluid Mech. 49, 249 (2017).

[5] S. Parsa and G. A. Voth, Inertial Range Scaling in Rotations of Long Rods in Turbulence, Phys. Rev. Lett. 112, 024501 (2014).

[6] M. Shin and D. L. Koch, Rotational and translational dispersion of fibres in isotropic turbulent flows, J. Fluid Mech. 540, 143 (2005).

[7] A. D. Bordoloi and E. Variano, Rotational kinematics of large cylindrical particles in turbulence, J. Fluid Mech. 815, 199 (2017).

[8] N. Pujara, T. B. Oehmke, A. D. Bordoloi, and E. A. Variano, Rotations of large inertial cubes, cuboids, cones, and cylinders in turbulence, Phys. Rev. Fluids 3, 054605 (2018).

[9] S. Bounoua, G. Bouchet, and G. Verhille, Tumbling of Inertial Fibers in Turbulence, Phys. Rev. Lett. 121, 124502 (2018).

[10] S. Kuperman, L. Sabban, and R. van Hout, Inertial effects on the dynamics of rigid heavy fibers in isotropic turbulence, Phys. Rev. Fluids 4, 064301 (2019).

[11] A. Pumir and M. Wilkinson, Orientation statistics of small particles in turbulence, New J. Phys. 13, 093030 (2011).

[12] S. Parsa, E. Calzavarini, F. Toschi, and G. A. Voth, Rotation Rate of Rods in Turbulent Fluid Flow, Phys. Rev. Lett. 109, 134501 (2012).

[13] L. Chevillard and C. Meneveau, Orientation dynamics of small, triaxial-ellipsoidal particles in isotropic turbulence, J. Fluid Mech. 737, 571 (2013).

[14] C. Brouzet, G. Verhille, and P. Le Gal, Flexible Fiber in a Turbulent Flow: A Macroscopic Polymer, Phys. Rev. Lett. 112, 074501 (2014). 
[15] A. Gay, B. Favier, and G. Verhille, Characterisation of flexible fibre deformations in turbulence, Europhys. Lett. 123, 24001 (2018).

[16] M. E. Rosti, A.A. Banaei, L. Brandt, and A. Mazzino, Flexible Fiber Reveals the Two-Point Statistical Properties of Turbulence, Phys. Rev. Lett. 121, 044501 (2018).

[17] S. Allende, C. Henry, and J. Bec, Stretching and Buckling of Small Elastic Fibers in Turbulence, Phys. Rev. Lett. 121, 154501 (2018).

[18] J. R. Picardo, R. Singh, S. S. Ray, and D. Vincenzi, Dynamics of a long chain in turbulent flows: Impact of vortices, Phil. Trans. R. Soc. A 378, 20190405 (2020).

[19] M. Sulaiman, E. Climent, B. Delmotte, P. Fede, F. Plouraboué, and G. Verhille, Numerical modeling of long flexible fibers in homogeneous isotropic turbulence, Eur. Phys. J. E 42, 132 (2019).

[20] R. Ni, S. Kramel, N. T. Ouellette, and G. A. Voth, Measurements of the coupling between the tumbling of rods and the velocity gradient tensor in turbulence, J. Fluid Mech. 766, 202 (2015).

[21] N. Pujara, G. A. Voth, and E. Variano, Scale-dependent alignment, tumbling and stretching of slender rods in isotropic turbulence, J. Fluid Mech. 860, 465 (2019).

[22] O. Cadot, D. Bonn, and S. Douady, Turbulent drag reduction in a closed flow system: Boundary layer versus bulk effects, Phys. Fluids 10, 426 (1998).

[23] A. D. Bordoloi, E. Variano, and G. Verhille, Lagrangian time scale of passive rotation for mesoscale particles in turbulence, Front. Mar. Sci. 7, 473 (2020).

[24] G. Verhille and A. Bartoli, 3d conformation of a flexible fiber in a turbulent flow, Exp. Fluids 57, 117 (2016).

[25] O. Faugeras and Q.-T. Luong, The Geometry of Multiple Images (The MIT Press, 2001).

[26] R. Hartley and A. Zisserman, Multiple View Geometry in Computer Vision, 2nd ed. (Cambridge University Press, Cambridge, 2003).

[27] A. Agrawal, S. Ramalingam, Y. Taguchi, and V. Chari, A theory of multi-layer flat refractive geometry, in IEEE Conference on Computer Vision and Pattern Recognition (IEEE, 2012), pp. 3346-3353.

[28] H. M. De La Rosa Zambrano, G. Verhille, and P. Le Gal, Fragmentation of magnetic particle aggregates in turbulence, Phys. Rev. Fluids 3, 084605 (2018).

[29] N. T. Ouellette, H. Xu, and E. Bodenschatz, A quantitative study of three-dimensional Lagrangian particle tracking algorithms, Exp. Fluids 40, 301 (2005).

[30] See Supplemental Material at http://link.aps.org/supplemental/10.1103/PhysRevFluids.xx.xxxxxx for download a video which illustrates the efficiency of the reconstruction algorithm by superimposing the projection of a reconstructed fiber after the different steps onto the experimental images.

[31] S. Douady, Y. Couder, and M. E. Brachet, Direct observation of the intermittency of intense vorticity filaments in turbulence, Phys. Rev. Lett. 67, 983 (1991).

[32] J. Jiménez and A. A. Wray, On the characteristics of vortex filaments in isotropic turbulence, J. Fluid Mech. 373, 255 (1998).

[33] T. Leung, N. Swaminathan, and P. A. Davidson, Geometry and interaction of structures in homogeneous isotropic turbulence, J. Fluid Mech. 710, 453 (2012).

[34] U. Frisch, Turbulence, the Legacy of A.N. Kolmogorov (Cambridge University Press, Cambridge, 1995).

[35] F. Moisy and J. Jiménez, Geometry and clustering of intense structures in isotropic turbulence, J. Fluid Mech. 513, 111 (2004).

[36] P. K. Yeung, X. M. Zhai, and K. R. Sreenivasan, Extreme events in computational turbulence, Proc. Natl. Acad. Sci. 112, 12633 (2015). 